\title{
New Anticorrosive Coating Compositions Based on 7- (2,3Dihydroxepropyl) Theophyline Modified Polyesteramide Resins as Binder for Industrial Applications
}

\author{
H. Abd El-Wahab" ${ }^{\#}$ F. Abdel-Hai, M. Mostafa ${ }^{*}$, A.M. Naser \\ and Z.I. Elbialy \\ Chemistry Department, Faculty of Science, Al Azhar \\ University, Cairo, Egypt and 'Department of Chemistry, \\ Faculty of Science, Jazan University, Jazan, KSA.
}

\begin{abstract}
JEW modified anti-corrosive polyesteramide resins were obtained 1 by condensation polymerization reaction between phthalic anhydride (PA) and N, N- bis - (2-hydroxyethyl) linseed oil fatty acid amide (HELA) which was partially replaced with 7(2,3dihydroxepropyl) theophyline as the ingredient source of the polyol used. The structure of the resin was confirmed by FT-IR spectral studies. The coatings of $40+/-5 \mu \mathrm{m}$ thickness were applied to the surface of glass panels and mild steel strips by means of a brush. The coating performance of the resins was evaluated using international standard test methods (ASTM) and involved the measurement of phyisco- mechanical properties such as viscosity, drying time, specular gloss, pencil hardness, adhesion, flexibility and impact resistance. Chemical resistance of the resins to water, acid, alkali and solvent was also evaluated to ascertain their suitability as a surface coatings vehicle. The results show that the modification enhances both phyisco- mechanical and chemical properties. The resins were incorporated within primer formulations and evaluated as anti-corrosive single coatings. The results illustrate that the introduction of 7- (2,3dihydroxepropyl)theophyline, containing a theophyline ring, within the resin structure, enhances the corrosion resistance performance of polyesteramide resins.
\end{abstract}

Keywords: Polyesteramide, 7-(2,3Dihydroxepropyl) Theophyline, Theophyline ring, Corrosion inhibition, Anticorrosive coating and Surface coating.

Polyesteramide resins (PEA) combine the advantageous properties of both polyester and polyamide resins, such as high melting temperature, fast crystallization, good mechanical properties, good solvent resistance and low water absorption ${ }^{(1-2)}$. The presence of repeating units of ester (-COOR) and amide (-NCOR) in the polymeric chain of polyesteramide improves the ease of

"Corresponding author:

E- mail: hamada19982000@ hotmail.com, Telephone: +20 107133466, Fax: +20 222629354 
application, thermal stability, chemical and water resistance, and also contributes to faster drying and enhanced hardness compared to normal alkyds ${ }^{(3-6)}$. Several polyesteramides have been synthesized from conventional and nonconventional seed oils to improve their drying ability, mechanical and corrosion protective efficiency ${ }^{(7-10)}$. The modification results in the formation of N, N-bis (2-hydroxyethyl) fatty acid amide (HEFA) monomer, which plays a vital role in the synthesis of organic polymers and in addition also finds application as a polymer cross- linker. Sharif Ahmad et al. (1999-2009) have successfully used it as a starting material for the development of polyetheramides, polyesteramides, polyesteramide urethanes and polyamide urethanes, which find application as protective coating materials and can be used as an effective antibacterial and biologically safe corrosion protective material ${ }^{(11-22)}$. Recently, new modified polyesteramide compositions were prepared and evaluated as anticorrosive varnish. The resin prepared by partial replacement of hydroxy ethyl fatty acid amide (HEFA) by polyethylene glycol (PEG) without affecting the resin constants. Primer formulations based on this resin showed good corrosion inhibiting properties. ${ }^{(23)}$. Also, new modified polyesteramide compositions were prepared and evaluated as vehicles for surface coating. This modification, as expected, improved the film performance and durability and lead to substantial antimicrobial growth control ${ }^{(24-25)}$. Blends of poly (vinyl chloride) (PVC) and polyesteramides were prepared to improve the notch impact strength of PVC ${ }^{(26)}$. Various modified polyesteramide varnishes as antimicrobial growth control were prepared and evaluated as new binder for organic surface coating (27). Polyimides, which belong to the class of heterocyclic polymers, have limited processing because fully cured imide polymers are generally infusible and insoluble in most solvents. To attain thermo-plasticity, polyimides have been modified either through the addition of a side chain or through the introduction of flexible, non-symmetrical and thermally stable linkages to the polymer backbone. Among these modifications, natural amino acids have been used for the preparation of polyimide with polyether, polyester or polyamides ${ }^{(28-31)}$. The aim of the present work was directed to characterize and evaluate new modified anti-corrosive polyesteramide resins for use in protective coating formulations. These modified resins were prepared by partial replacement of N, N- bis - (2hydroxyethyl) linseed oil fatty acid amide (HELA) with 7- (2,3dihydroxepropyl) theophyline as a new source of the polyol source.

\section{Experimental}

\section{Materials}

The linseed oil fatty acid (LOFA) used was supplied by Echantillon, Belgium, the phthalic anhydride (PA) by Avondate Laboratories, England and the diethanolamine (DEA) and - (2,3dihydroxepropyl) theophyline were obtained from Sigma Aldrish Germany, sulphuric acid, anhydrous sodium carbonate, benzene, glacial acetic acid and ethyl acetate were obtained from El Nasr Pharmaceutical Company, Egypt. The xylene and mineral turpentine products were obtained from Misr Petroleum Company, Egypt.

Egypt. J. Chem. 54, No. 2 (2011) 


\section{Methods and techniques}

Synthesis of $N$, $N$-bis (2-hydroxyethyl) linseed oil fatty acid amide (HELA)

A mixture of freshly distilled diethanolamine (DEA) $(11.55 \mathrm{~g}, 0.11 \mathrm{~mol})$ and linseed oil fatty acid $(28.0 \mathrm{~g}, 0.1 \mathrm{~mol})$ was added to a $250 \mathrm{ml}$ round-bottom flask fitted with a Dean and Stark trap. The mixture was allowed to reflux in the presence of $15 \%$ xylene until the theoretical amount of water $(1.8 \mathrm{ml}, 0.1 \mathrm{~mol})$ was collected, indicating the complete formation of $\mathrm{N}, \mathrm{N}$-bis (2-hydroxyethyl) linseed oil fatty acid amide (HELA). resins

Synthesis of polyesteramide and new modified anti-corrosive polyesteramide

Polyesteramide resins were prepared through a condensation polymerization reaction via a solvent process, in a one step reaction. A mixture of a calculated amount of hydroxy ethyl linseed oil fatty acid amide (HELA), replaced partially with 7-(2,3dihydroxepropyl )theophyline as the ingredient source of the polyol and phthalic anhydride (PA) - as the source of the polybasic acid ingredient, was added to a $250 \mathrm{ml}$ round-bottom flask fitted with a Dean and Stark trap and refluxed in the presence of $10 \%$ xylene. The course of esterification was followed by observing the theoretical amount of water liberated. The resins were prepared, covering a wide range of oil lengths and hydroxyl content $(0,10,20$ and $30 \%$ excess-OH). It should be noted that within each set of formulations the total number of acid and hydroxyl equivalent for the various runs were kept constant ${ }^{(32)}$. Resin characteristic constant and weight changes of the various formulations are illustrated in Tables 1 and 2. Polyester amide calculations can be used to predict the completed formulation, properties of the resin, the amount of reaction water liberated and the probable risk of gelation. The calculation of water evolved is also useful both as a tool for following the course of esterification reaction and to find the theoretical yield.

\section{Measurements}

The structure of the prepared 7-(2,3dihydroxepropyl )theophyline was confirmed by the melting point. The structure of the prepared N, N-bis (2hydroxyethyl) linseed oil fatty acid amide (HELA) was confirmed by acid value (ASTM D 1639-96) and hydroxyl value (ASTM D 1957- 01). Infrared spectra of the prepared polyesteramide resin was recorded in polymer/ $\mathrm{KBr}$ pellets using Shimadzu FT-IR 400 spectrometer was also undertaken for confirmation of the polyesteramide resin structure as showed in Fig. 1and tabulated in Table 3. The colour (ASTM D 1544-04) of the prepared polyesteramide and the new modified anti-corrosive polyesteramides resins was undertaken using the 'Gardner 1953 standard colour', whereas the viscosity (ASTM D 1545-07) was established by the use of Gardner -Holdt viscosity tubes. 


\section{Preparation of polyesteramide coatings}

The solid content (ASTM D1644-01) of the modified and unmodified polyesteramide resins were adjusted by means of thinning with mineral turpentine to $50 \%$ solids for the $0,10,20$ and $30 \%$ excess $-\mathrm{OH}$ samples. The resin samples were then filtered and the driers combination added $(\mathrm{Co}, \mathrm{Zr}$ and $\mathrm{Ca}$ Octoates, $0.04,1.0$ and $0.05 \%$ based on metal / solid resin, respectively). The resin material was then applied onto both glass panels (ASTM D3891-02) and steel strips (ASTM D609 - 00) at room temperature by means of a brush.

TABLE 1. Resin constants for theophyline -modified polyesteramides.

\begin{tabular}{|c|c|c|c|c|c|c|c|c|c|c|}
\hline $\begin{array}{c}\text { Resin } \\
\text { No. }\end{array}$ & $\begin{array}{c}\text { Excess - } \\
\text { OH \% }\end{array}$ & Ingredients & $\mathbf{E}$ & $\mathbf{F}$ & $\mathbf{e}_{0}$ & $\mathbf{e}_{\mathrm{A}}$ & $\mathbf{e}_{B}$ & $\mathbf{R}$ & $\mathbf{K}$ & $\begin{array}{c}\mathrm{H}_{2} \mathrm{O} \text { off } \\
\mathrm{ml}\end{array}$ \\
\hline$I_{a-d}$ & 0 & $\begin{array}{c}\text { HELA } \\
\text { DHTP } \\
\text { PA }\end{array}$ & $\begin{array}{r}184 \\
127 \\
74.1\end{array}$ & 2 & 0.520 & 0.260 & 0.260 & 1.00 & 1.00 & 2.3 \\
\hline $\mathrm{II}_{\mathrm{a}-\mathrm{d}}$ & 10 & $\begin{array}{c}\text { HELA } \\
\text { DHTP } \\
\text { PA }\end{array}$ & $\begin{array}{r}184 \\
127 \\
74.1\end{array}$ & 2 & 0.573 & 0.273 & 0.300 & 1.10 & 1.05 & 2.5 \\
\hline III $_{a-d}$ & 20 & $\begin{array}{c}\text { HELA } \\
\text { DHTP } \\
\text { PA }\end{array}$ & $\begin{array}{l}184 \\
127 \\
74.1\end{array}$ & 2 & 0.656 & 0.298 & 0.358 & 1.20 & 1.10 & 2.7 \\
\hline $\mathrm{IV}_{\mathrm{a}-\mathrm{d}}$ & 30 & $\begin{array}{c}\text { HELA } \\
\text { DHTP } \\
\text { PA }\end{array}$ & $\begin{array}{l}184 \\
127 \\
74.1\end{array}$ & 2 & 0.778 & 0.339 & 0.439 & 1.30 & 1.15 & 3.1 \\
\hline
\end{tabular}

HELA : Hydroxy Ethyl Linseed Amide

PA : Phthalic Anhydride

DHTP : Hydroxypropyl theophyline

\footnotetext{
$\underline{\mathbf{E}}$ : Equivalent Weight $\underline{\mathbf{e}}_{\mathbf{A}}$ : Number of acid equivalent $\underline{\mathbf{e}}_{\mathbf{B}}$ : Number of hydroxyl equivalent

$\underline{\mathbf{e}}_{\mathbf{0}}$ : Total equivalent present at the start of the reaction

$\underline{\mathbf{F}}:$ Functionality $\quad \underline{\mathbf{K}}:$ Alkyl constant $\left(\mathrm{m}_{0} / \mathrm{e}_{\mathrm{A}}\right)$

R : Ratio of total-OH groups to total-COOH groups $\left(\mathrm{e}_{\mathrm{B}} / \mathrm{e}_{\mathrm{A}}\right)$
} 
TABLE 2. List of hydroxyl equivalent of different runs.

\begin{tabular}{|c|c|c|}
\hline Resin & HELA & Theophyline \\
\hline $\mathrm{a}$ & 1.00 & 0.00 \\
$\mathrm{~b}$ & 0.90 & 0.10 \\
$\mathrm{c}$ & 0.80 & 0.20 \\
$\mathrm{~d}$ & 0.70 & 0.30 \\
\hline
\end{tabular}

Film casting and testing

Glass plates $(100 \times 150 \mathrm{~mm})$ coated with the individual resins were utilized to measure the drying schedule, in terms of time (touch dry - through dry) (ASTM D1640-03). In addition, the same coated glass plates were also used to measure the degree of gloss for individual resin coatings (ASTM 523-08). The measurements were undertaken using a gloss meter set at a $20^{\circ}$ angle. The chemical resistance (ASTM D 870-02, D 2792-04) of the dried resin coatings to different solvent media was undertaken on glass panels $(25 \times 75 \mathrm{~mm})$. The coated steel strips were utilized to measure the coating film thickness (ASTM D 1005- 07), with this being undertaken using a Model 2100 Minitest microprocessor coating thickness gauge, manufactured by Electro - Physic, West Germany. The thickness of the coatings was found to be $40+/-5 \mu \mathrm{m}$. The coated steel strips were also used also to measure the mechanical resistance of the coated resin films, with testing undertaken on adhesion strength, by means of the cross-cut method (ASTM D 3359-02), flexibility (ASTM D 522-93), pencil hardness (ASTM D 3363-00) and resistance to mechanical damage (impact resistance) (ASTM D 2794-04).

Anti-corrosive performance evaluation of primer formulations through accelerated testing

A salt fog chamber was utilized for each of the resin formulations; with a set of three coated panels being placed in the salt spray chamber according to ASTM B117-03. The corrosion resistance was evaluated in terms of blistering, scribe failure and degree of rusting, in relation to ASTM standards, i.e. ASTM D71402, D1654-00 and D610-01, respectively.

\section{Results and Discussion}

In the recent years there has been considerable interest in improving polyesteramide resins. A number of polyesteramide have been developed by the reaction of linseed oil fatty amide with dicarboxylic acids/anhydrides ${ }^{(23-25)}$. In the present work, the newly modified anti-corrosive polyesteramides resins were obtained by partial replacement of hydroxyethyl linseed oil fatty acid amide (HELA) with 7-(2,3dihydroxepropyl) theophyline as the ingredient source of the polyol. The reaction of LOFA with DEA to give HELA is demonstrated in Scheme 1 , which was confirmed by acid and hydroxyl value. The measured acid 
value was $6 \mathrm{mg} \mathrm{KOH} / \mathrm{g}$, whilst the theoretical value is $0 \mathrm{mg} \mathrm{KOH} / \mathrm{g}$, and the measured hydroxyl value was $308 \mathrm{mg} \mathrm{KOH} / \mathrm{g}$ whilst the theoretical value is $305.72 \mathrm{mg} \mathrm{KOH} / \mathrm{g}$, which indicates the complete formation of HELA.<smiles>[R]C(=O)N[C@@H](CO)[13CH](CO)[14CH2]O</smiles>

LOFA DEA HELA

Scheme 1. Synthesis of N,N-bis(2-hydroxyethyl) linseed oil fatty acid amide(HELA) .

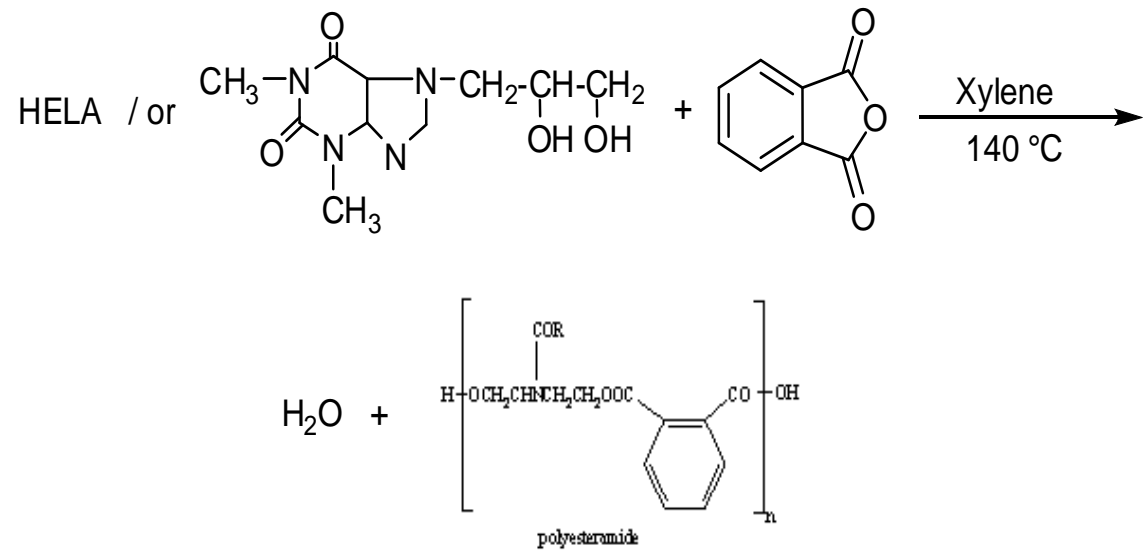

Scheme 2. Synthesis of theophyline -modified polyesteramides .

R : Theophyline ring

HELA : N, N-bis (2-hydroxyethyl) linseed oil fatty acid amide

Spectral analysis of polyesteramide resin

Polyesteramide resins were prepared by the reaction of HELA or 7(2,3dihydroxepropyl )theophyline with phthalic anhydride (PA) in the presence of xylene, as illustrated in Scheme 2 . It contains both ester and amide linkages in the polymer backbone. This has been confirmed by both FT-IR spectral analysis, as shown in Fig. 1. The key peaks of the IR spectra of polyesteramide are shown in Table 3, which indicates the presence of both ester and amide groups, confirming the formation of polyesteramide. 


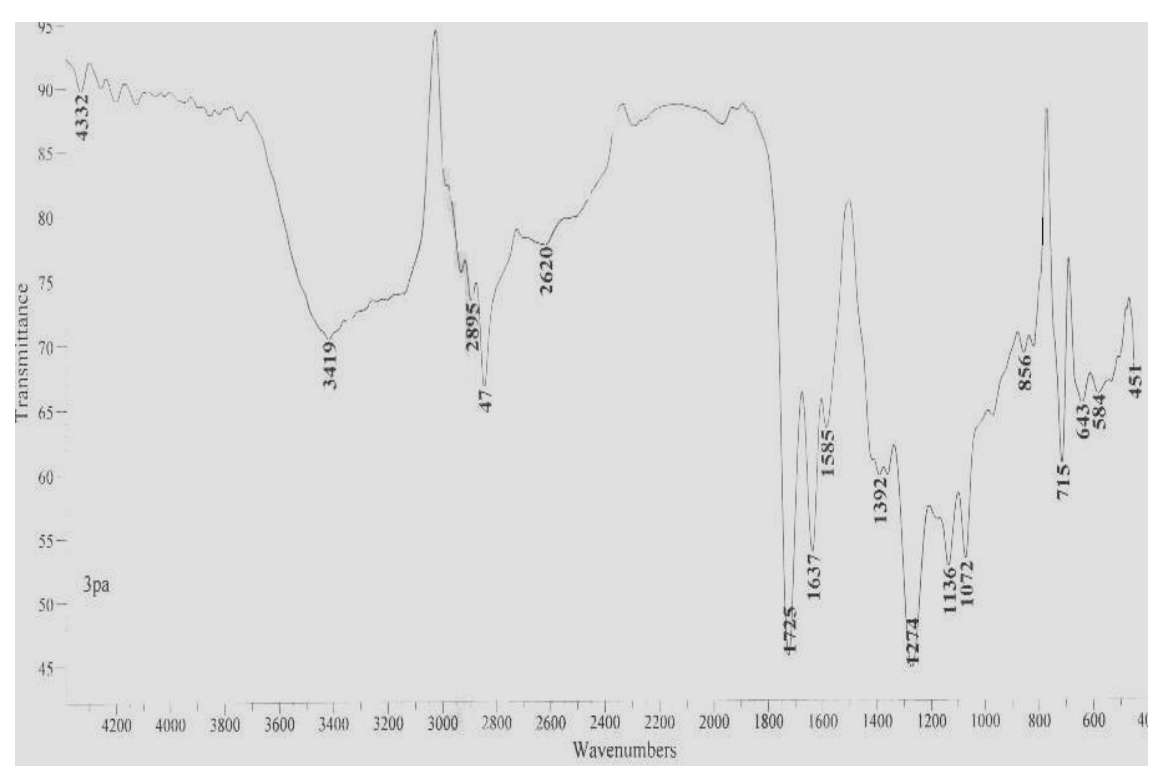

Fig.1. I.R Spectrum of [DHTP] modified polyesteramide resin.

TABLE 3. FTIR spectra of polyesteramide resin.

\begin{tabular}{|l|c|}
\hline Functional group & IR peak $\left(\mathbf{c m}^{-1}\right)$ \\
\hline $\mathrm{OH}$ & 3419 \\
\hline $\mathrm{CON}$ amide carbonyl & 1637 \\
\hline $\mathrm{COO}$ ester carbonyl & 1725 \\
\hline $\mathrm{C}-\mathrm{N}$ & 1455 \\
\hline $\mathrm{CH}_{2}$ symmetric & 2895 \\
\hline $\mathrm{CH}_{2}$ a symmetric & 2847 \\
\hline Ring stretching vibration of aromatic nuclei & 715 \\
\hline
\end{tabular}

Physical-mechanical characterization of the new modified anti-corrosive polyesteramide resin

During the course of the preparation of the resin, the amount of collected water present in the trap was observed against the reaction time (Fig. 2). The effect of the 7-(2,3dihydroxepropyl) theophyline on the physical properties of the resin i.e. colour, viscosity, air drying time, are shown in Table 4 . The effect on the mechanical properties such as gloss, flexibility (bend), adhesion, scratch hardness and resistance to mechanical damage (impact resistance) are represented in Table 5. The results tabulated in Table 5 show that the polyesteramide, and the new modified anti-corrosive polyesteramide compositions, give a very clear transparent and homogenous appearance. The 
figure also shows that the reaction time is marginally increased with an increase in the amount of 7-(2,3dihydroxepropyl) theophyline, but excess hydroxyl content leads to a decrease in the reaction time. The colours of resins are observed to be dark brown due to the presence of nitrogenous base and the viscosity of resins decrease with an increase in the percentage of both 7 (2,3dihydroxepropyl ) theophyline and excess hydroxyl content. The drying time is observed to be proportional with both 7-(2,3dihydroxepropyl ) theophyline and excess hydroxyl content, the drying time was decreased with increasing the percent of 7-(2,3dihydroxepropyl) theophyline as the modifier. The mechanical characteristics of the various modified resins tabulated in Table 5. The results indicate that the modified films show outstanding performance, compared to unmodified films, the gloss results for the resins increases through modification, which may be attributed to the incorporation of dihydroxyl polyol containing a theophyline ring within the resin structure. The presence of the repeating units of ester (-COOR) in the polymeric chain of polyesteramide improves the ease of application, as well as enhancing the hardness and impact resistance properties. The flexibility test for the coated panel, carried out at the mandrel diameter of $6 \mathrm{~mm}$, shows no evidence of cracks or de-laminating of the coating film. This high flexibility of the coating is attributed to the resin being composed of a polyester backbone. The cross-hatch test, carried out to determine the adhesion of the coating, clearly indicates that the modified coating has very good adhesion properties. No de-laminating or detachment, of coatings at the edges, and within the square lattice, was observed. It is clear that the ability of the coating on the pre- treated mild steel substrate, in terms of preventing water ingress, is good, which is one of the contributing factors for the improved adhesion of the coating to the substrate. Improvement of the mechanical properties appears to be due to the amide groups, which results in poly (amide), containing a theophyline ring within the resin structure.

Chemical resistance of the new modified anti-corrosive polyesteramide resin

The chemical resistances testing of the new modified anti-corrosive polyesteramide resins were undertaken on glass panels $(25 \times 75 \mathrm{~mm})$. The resin coated glass panels were sealed using paraffin wax on the edges of the panels. The samples were immersed to half their length in the various test solutions (water, $5 \%$ by weight anhydrous sodium carbonate, $10 \%$ by weight sulphuric acid, and benzene / mineral turpentine solvent mixture (1:3 by volume). The panels were then removed from the solution, wiped carefully and allowed to dry at room temperature, prior to testing for any change. The obtained data is tabulated in Table 5. The data indicates that an increase in the levels of 7 (2,3dihydroxepropyl) theophyline lead to a substantial improvement in the films resistance towards water, alkali and acid. This may be attributable to the introduction of the theophyline ring within the resin structure. However, the 7(2,3dihydroxepropyl) theophyline does not appear to affect the solvent resistance properties, since all the examined films showed excellent performance within the experimental time of 30 days. In addition, it was observed that the stove dried films showed enhanced characteristics compared to the air dried films.

Egypt. J. Chem. 54, No. 2 (2011) 
New Anticorrosive Coating Compositions ... 
Corrosion resistance of the new modified anti-corrosive polyesteramide resin

The research work was extended to study the utilization of the new modified anticorrosive polyesteramides as a binder in primer formulations. Primer formulations based on unmodified and modified polyesteramide were prepared, with the various formulations represented in Table 6. The primer formulations were evaluated on coated steel panels. To ensure that the steel panels were free of all surface contamination before the coating application, pre- treatment involved wire brushing and sand paper cleaning of the surface. The coated steel panels were exposed to salt spray (fog) corrosion cabinet manufactured by Sheen Instruments Limited, England, for $500 \mathrm{hr}$. Photographic reference standards were used to evaluate the degree of blistering. The reference standards highlight the various degrees of blistering that can potentially develop when paint systems are subjected to these specific test conditions. The results of salt spray test are represented in Table 7 . Figure 4 shows that there are significant differences between the tested formulations, which depend upon the amount of modifier present 7-(2,3dihydroxepropyl) theophyline. The anti-corrosive performance of coatings containing the modifier, improves with increased levels of the modifier. The improvement in corrosion resistance may be attributed to the introduction of a compound that contains amide and imide linkages, which results in poly (amide), containing a theophyline ring within the resin structure. Also, the high molecular weight of the modifier decreases the permeability of coating to water, oxygen and the aggressive ions such as chloride. The corrosion resistance increase is also due to the absorption of resin onto the metal through its hydroxyl function.

TABLE 4. Varnishes characteristics data .

\begin{tabular}{|c|c|c|c|c|c|c|}
\hline $\begin{array}{c}\text { Resin } \\
\text { No. }\end{array}$ & $\begin{array}{c}\text { Excess- } \\
\text { OH \% }\end{array}$ & $\begin{array}{l}\text { Replacement } \\
\text { \% of DHTP }\end{array}$ & $\begin{array}{l}\text { Reaction } \\
\text { time (min.) }\end{array}$ & $\begin{array}{c}\text { Color } \\
\text { (Gardner) }\end{array}$ & $\begin{array}{c}\text { Viscosity } \\
\text { (poise) }\end{array}$ & $\begin{array}{c}\text { Air drying } \\
\text { time } \\
\text { (min)V }\end{array}$ \\
\hline Ia & \multirow{4}{*}{0} & 0 & 50 & 8 & 23 & $4 \mathrm{hr}$ \\
\hline $\mathrm{Ib}$ & & 10 & 54 & 11 & 20.3 & 30 \\
\hline Ic & & 20 & 57 & 12 & 20.4 & 35 \\
\hline Id & & 30 & 58 & 12 & 21.2 & 35 \\
\hline IIa & \multirow{4}{*}{10} & 0 & 52 & 8 & 23.5 & $4.50 \mathrm{hr}$ \\
\hline $\mathrm{IIb}$ & & 10 & 55 & 12 & 21.4 & 30 \\
\hline IIc & & 20 & 57 & 12 & 21.6 & 30 \\
\hline IId & & 30 & 60 & 13 & 21.8 & 30 \\
\hline IIIIa & \multirow{4}{*}{20} & 0 & 55 & 9 & 24 & $6.5 \mathrm{hr}$ \\
\hline IIIlb & & 10 & 56 & 13 & 22 & 30 \\
\hline IIIC & & 20 & 59 & 14 & 22.1 & 25 \\
\hline IIII & & 30 & 62 & 14 & 22.3 & 25 \\
\hline IVa & \multirow{4}{*}{30} & 0 & 56 & 10 & 24.5 & 6 \\
\hline $\mathrm{IVb}$ & & 10 & 58 & 14 & 22.3 & 20 \\
\hline IVc & & 20 & 64 & 14 & 22.6 & 20 \\
\hline IVd & & 30 & 66 & 14 & 22.6 & 15 \\
\hline
\end{tabular}

Egypt. J. Chem. 54, No. 2 (2011) 
TABLE 7. Evaluation the corrosion resistance of primer formulations.

\begin{tabular}{|c|c|c|c|c|}
\hline \multirow{2}{*}{$\begin{array}{c}\text { Formulation } \\
\text { No. }\end{array}$} & \multicolumn{2}{|c|}{ Blistering } & \multirow{2}{*}{$\begin{array}{l}\text { Scribe Failure } \\
\text { (mm) }\end{array}$} & \multirow{2}{*}{ Rust grade } \\
\hline & Size & Frequency & & \\
\hline Ia & 6 & $\mathrm{D}$ & 9 & 5 \\
\hline $\mathrm{Ib}$ & 7 & $\mathrm{D}$ & 7 & 6 \\
\hline Ic & 7 & MD & 6 & 6 \\
\hline Id & 8 & $\mathrm{M}$ & 5 & 7 \\
\hline IIa & 6 & $\mathrm{D}$ & 9 & 5 \\
\hline $\mathrm{IIb}$ & 7 & MD & 6 & 7 \\
\hline IIc & 8 & $\mathrm{M}$ & 5 & 7 \\
\hline IId & 9 & $\mathrm{~F}$ & 4 & 8 \\
\hline IIIa & 7 & MD & 8 & 5 \\
\hline IIIb & 8 & $\mathrm{M}$ & 6 & 7 \\
\hline IIII & 8 & $\mathrm{M}$ & 5 & 8 \\
\hline IIId & 9 & $\mathrm{~F}$ & 4 & 9 \\
\hline IVa & 7 & MD & 8 & 6 \\
\hline $\mathrm{IVb}$ & 9 & $F$ & 4 & 8 \\
\hline IVc & 9 & $\mathrm{~F}$ & 3 & 9 \\
\hline IVd & 9 & $\mathrm{~F}$ & 3 & 9 \\
\hline
\end{tabular}

The blistering size is graded from 10 to 0 , where 10 represents of no blistering and 0 representative of the largest blister. Blistering frequency is denoted by F, M, MD and D (few, medium, medium dense and dense).Painted, or coated, specimens subjected to a corrosive environment are also evaluated by recording the average (mean) maximum and minimum creepage from the scribe mark. Scribe failure is also rated on a scale from 10 to 0 , with 10 being zero $(\mathrm{mm})$ from the scribe mark and 0 is $16(\mathrm{~mm})$ from the scribe mark. Finally visual comparison of the surface with photographic reference standards, to determine the percentage of the area that has been rusted, is also used. These visual standards were developed in cooperation with the Steel Structure Painting Council (SSPC) to further standardization of test methods. The amount of rusting beneath, or through, a paint film is a significant factor in determining whether a coating system should be repaired, or replaced. The rust grade is rated on scale from 10 to 0 , where 10 is no rusting and 0 is severe rusting. 


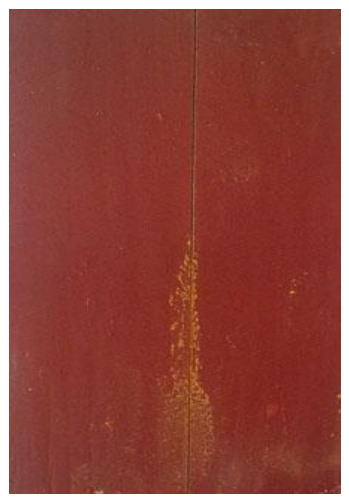

Blank

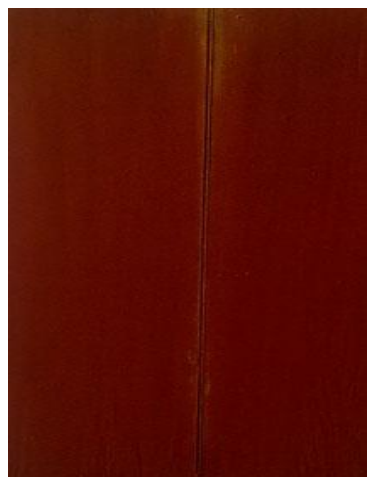

$20 \% \mathrm{EX}$

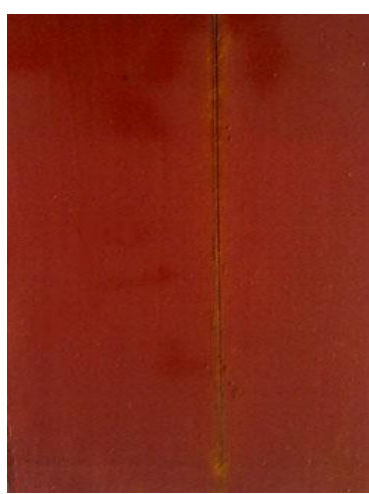

$0 \% \mathrm{Ex}$

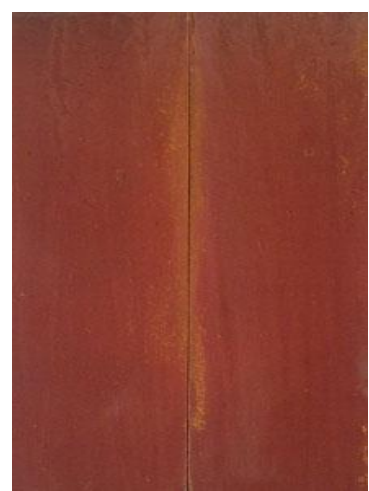

$10 \% \mathrm{Ex}$

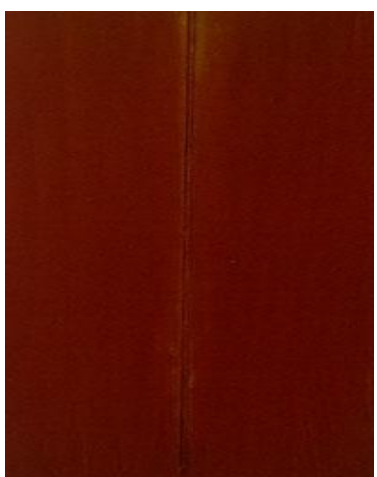

30\% EX

Fig. 3. Corrosion resistance of painted films.

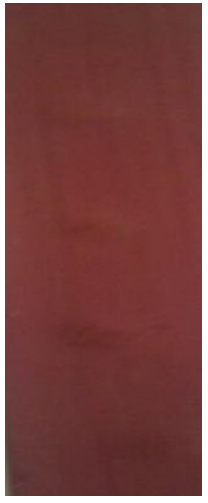

Blank

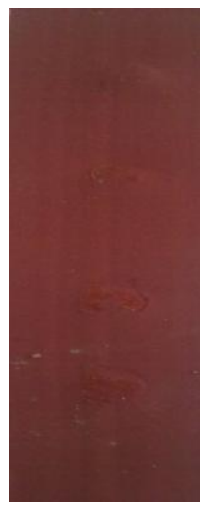

$\mathrm{O} \% \mathrm{Ex}$

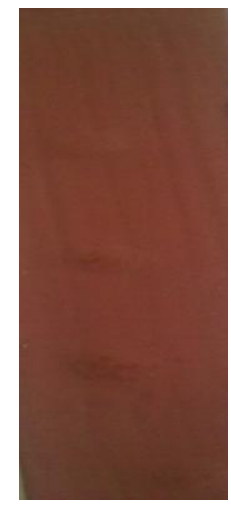

$10 \% \mathrm{Ex}$

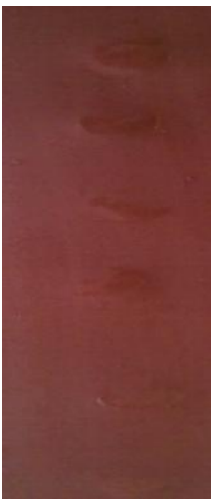

$20 \%$ Ex

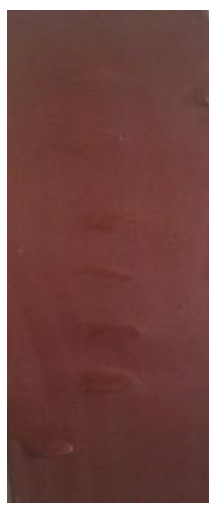

$30 \% \mathrm{Ex}$

Fig. 4. Impact resistance of painted films.

Egypt. J. Chem. 54, No. 2 (2011) 


\section{Conclusion}

We have successfully prepared modified polyesteramide resin by the partial replacement of hydroxy ethyl linseed oil fatty acid amide (HELA) with 7(2,3dihydroxepropyl )theophyline as the ingredient source of the polyol with phthalic anhydride [PA] as dibasic acid source, where the time of reaction and drying time are the only minor drawbacks. The modified resin shows enhanced physico- mechanical properties such as gloss, adhesion, scratch hardness and resistance to mechanical damage. It is also observed that the modified resin shows better film performance in terms of chemical resistance to water, acid and alkali. The corrosion resistance of the modified theophyline resins, incorporated within primer formulations, also improves with increased levels of the modifier. This improvement may be attributed to (i) the combination of amide and imide groups, which results in poly (amide), which containing a theophyline ring within the resin structure; (ii) the presence of repeating units of ester and amide in the polymeric chain of the modified polyesteramide; and (iii) the high molecular weight of the modifier decreases the permeability of the coating to water, oxygen and the aggressive ions such as chloride.

\section{References}

1. Bori, C. Sorta, and E. Zotteri, L., Transitions of a series of regular alternating linear polyesteramides. Polymer, 16 (8), 565-568. (1975)

2. Van Hutten, P.F., Magnus, R.M. and Gaymans, R.J., Segmented copolymers with polyesteramide units of uniform length: structure analysis. Polymer, 34(20), 4193 $4202(1993)$

3. Gast, L.E., Schneider, W.J. and Cowan, J.C., Polyesteramides from linseed oil for protective coatings. Journal of the American Oil Chemists' Society (JAOCS), 43(6), 418-421 (1966)

4. Gast, L.E., Schneider, W.J. and Cowan, J.C., Polyesteramides from linseed oil for protective coatings low acid- value polymers. Journal of the American Oil Chemists Society (JAOCS), 45 (7), 534-536 (1968).

5. Gast, L.E., Schneider, W.J., Mc Manis, G.E. and Cowan, J.C., Polyesteramides from linseed and soybean oils for protective coatings: Diisocyanate-modified polymers. Journal of the American Oil Chemists' Society (JAOCS), 46 (7), 360-364 (1969)

6. Economy, J., Liquid crystalline aromatic polyesters. Journal of Macromolecular Science, Part A: Pure and Applied Chemistry, 21,1705-17240 (1984)

7. Mahopatra, S.S. and Karak, N., Synthesis and characterization of polyesteramide resins from Nahar seed oil for surface coating applications. Progress in Organic Coatings, 51 (2), 103-108(2004) 
8. Shende, P.G., Jadhav, A.B. and Dabhade, S.B., Polyesteramide resins from dehydrated castor oil and various dibasic acids. Pigment \& Resin Technology, 31(5), 310-314 (2002)

9. Shende, P., Dabhade, S. and Kukarni, M., Soybean oil modified polyesteramide resins. Pigment \&Resin Technology, 32(1), 4-9. (2003)

10. Mistry, S. and Agarwal, D., Studies on the film characteristics of polyesteramide resins based on hydroxyl ethyl fatty acid amide. Pigment \&Resin Technology, 38(6), 366-371 (2009)

11. Ahmad, S., Naqvi, F., Verma, K. and Yadav, S., Studies on a newly developed linseed oil based alumina filled polyesteramide anticorrosive coating. Journal of Applied Polymer Science, 72 (13), 1679-1687 (1999)

12. Ahmad, S., Ashraf, S.M., Hasnat, A., Yadav, S. and Jamal, A., Studies on urethane- modified Alumina - filled polyesteramide coatings cured at ambient temperature. Journal of Applied Polymer Science, 82 (8), 1855 -1865 (2001)

13. Ahmad, S., Ashraf, S.M., Naqvi, F., Yadav, S. and Abu Hasnat, A., polyesteramide from Pongamia glabra oil for biologically safe anticorrosive coating. Progress in Organic Coatings, 47 (2), 95-102 (2003)

14. Zafar, F., Sharmin, E., Ashraf, S. M. and Ahmad, S., Studies on poly (stryne- comalic anhydride) modified polyesteramide based anticorrosive coating synthesized from a sustainable resources . Journal of Applied Polymer Science, 92 (4), 2538-2544 (2004)

15. Alam, M., Sharmin, E., Ashraf, S.M. and Ahmad, S., newly developed urethane modified polyetheramide-based anticorrosive coatings from a sustainable resource. Progress in Organic Coatings, 50(4) , 224-230 (2004)

16. Zafar, F., Ashraf, S.M. and Ahmad, S., Air drying polyesteramide from a sustainable resource. Progress in Organic Coatings, 51 (3), 250-256 (2004)

17. Zafar, F., Sharmin, E., Ashraf, S.M. and Ahmad, S., Ambient -cured polyesteramide- based anticorrosive coatings from linseed oil- A sustainable resource. Journal of Applied Polymer Science, 97(5), 1818-1824 (2005)

18. Zafar, F., Ashraf, S.M. and Ahmad, S., Studies on zinc- containing linseed oil based polyesteramide. Reactive and Functional Polymers, 67(10), 928-935 (2007)

19. Zafar, F., Ashraf, S.M. and Ahmed, S., Cd and Zn-incorporated polyesteramide coating materials from seed oil-A renewable resource. Progress in Organic Coatings, 59 (1), 68-75 (2007)

20. Ahmad, S., Ashraf, S.M. and Zafar, F., Development of linseed oil based polyesteramide without organic solvent at lower temperature. Journal of Applied Polymer Science, 104 (2), 1143-1148 (2007)

21. Zafar, F., Ashraf, S.M. and Ahmad, S., Development of Zn / Cd - incorporated poly (esteramide-urethane) from sustainable resources. Journal of Applied Polymer Science, 110 (1), 584-593 (2008)

Egypt. J. Chem. 54, No. 2 (2011) 
22. Alam, M., Ray, A. R. and Ahmad, S., Synthesis and characterization of poly (esteramide - urethane) from linseed oil as anticorrosive coatings. Journal of Applied Polymer Science, 114 (5), 3268-3273 (2009)

23. Salem A., Abd El-Wahab, H., Abdulsalam M., Abd El-Hai, F. and Abd ElFattah, M., New modified polyesteramide resin for industrial applications. Journal of Progress in Organic Coatings, 68, 219-224 (2010)

24. Abd El-Wahabb, H., Various modified polyesteramide varnishes as antimicrobial surface coating vehicles. Al-Azhr Bull. Sci. J. 19 (1) pp. 322-332 (jun 2008)

25. Ibrahim S., Abd El-Hai., F. and Gabr. M.Y., Antimicrobial activity of some modified polyesteramide resins for surface coatings. Al -Azhr Bull.Sci.J.2(Dec), (2010)

26. Kalouskova, R., Markvart, L., Kredatusova, J. and Brozek, J., Properties of poly (vinyl chloride) and polyesteramides blends. Journal of Applied Polymer Science, 114(2), 826-833. (2009)

27. Mostafa, M., Naser, A.M., Abdel-Hai F. and Abd El-Wahab. H., Various modified polyesteramide Varnishes as antimicrobial growth control. Al -Azhr Bull.Sci. J. 2 (Dec), (2009)

28. Iwakura, Y. and Yang, C. P., Keikichi Synthesis and properties of new aromaticaliphatic copolyimides. Macromolecular Chemistry, 175 (1), 137-159 (1974)

29. Yang, C. P., Synthesis of polymers containing pyromellitdiimide and benzimidazole or pyrrolobenzimidazole moieties and of their corresponding model compounds. Macromolecular Chemistry, 187 (11), 2509-2523 (1986)

30. Orzeszko, A. and Mirowski, K., Synthesis and structural studies of new copolyimides, influence of pyromellitimide rings on copolymer structure. Macromolecular Chemistry, 192 (8), 1841-1848 (1992)

31. Tomida, M., Nakato, T., Kuramochi, M., Shibata, M., Matsunami, S. and Kakuchi, T., Novel method of synthesizing poly (succinimide) and its copolymeric derivatives by acid catalysed polycondensation. Polymer, 37 (19), 4435-4437 (1996)

37. Patton, T.C., Alkyd Resin Technology, Formulating and Calculations, John Wiley and Sons, New York (1962)

(Received 31/5/2011;

accepted 4/9/2011) 


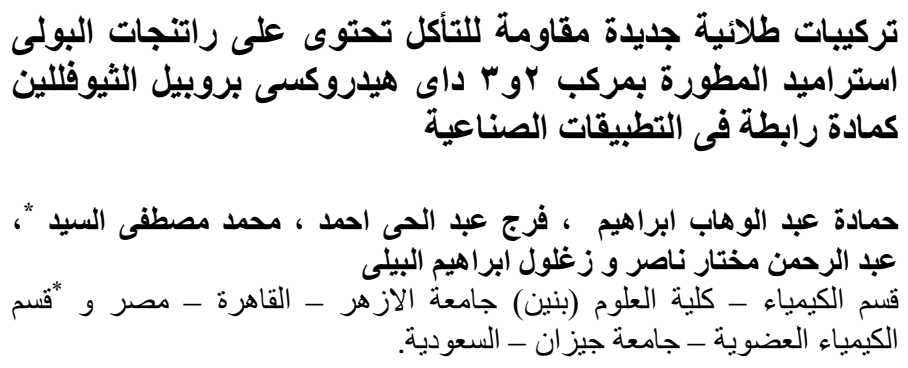

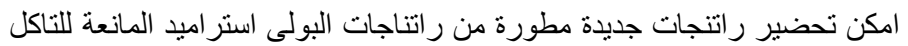

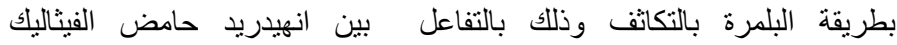

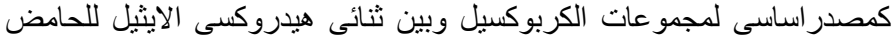

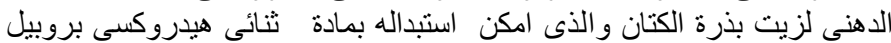

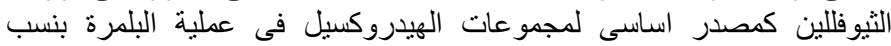
مختلفة.

وقد نم التأكد من تحضير الراتتج المطور للبولى استراميد باستخدام الاشعة الردام

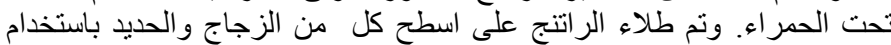

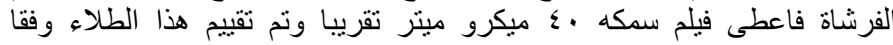

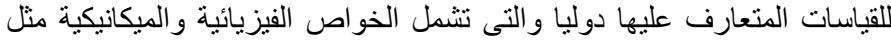

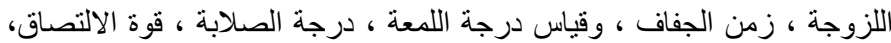
المرونة وايضا مقاومة الدهان الدان للصدمة.

وكذلك تم تقييم المقاومة الكيميائيه للر اتتج لكل من الماء و الاحماض و القلويات

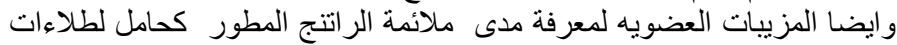

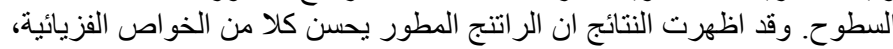
الميكانيكية وايضا الكيميائيه.

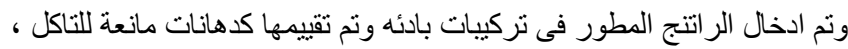

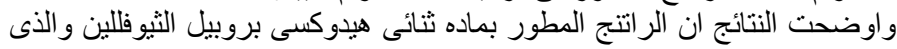

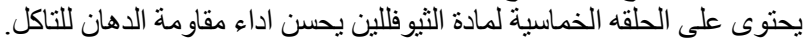

